\title{
Automated Assume-Guarantee Reasoning for Omega-Regular Systems and Specifications
}

\author{
Sagar Chaki Arie Gurfinkel \\ Software Engineering Institute, Carnegie Mellon University
}

\begin{abstract}
We develop a learning-based automated Assume-Guarantee (AG) reasoning framework for verifying $\omega$-regular properties of concurrent systems. We study the applicability of non-circular (AGNC) and circular (AG-C) AG proof rules in the context of systems with infinite behaviors. In particular, we show that AG-NC is incomplete when assumptions are restricted to strictly infinite behaviors, while AG-C remains complete. We present a general formalization, called LAG, of the learning based automated AG paradigm. We show how existing approaches for automated AG reasoning are special instances of LAG. We develop two learning algorithms for a class of systems, called $\infty$-regular systems, that combine finite and infinite behaviors. We show that for $\infty$-regular systems, both AGNC and AG-C are sound and complete. Finally, we show how to instantiate LAG to do automated AG reasoning for $\infty$-regular, and $\omega$-regular, systems using both AG-NC and AG-C as proof rules.
\end{abstract}

\section{Introduction}

Compositional reasoning $[8,13]$ is a widely used technique for tackling the statespace explosion problem while verifying concurrent systems. Assume-Guarantee (AG) is one of the most well-studied paradigms for compositional reasoning $[19,14]$. In AG-style analysis, we infer global properties of a system from the results of local analysis on its components. Typically, to analyze a system component $C$ locally, we use an appropriate "assumption", a model of the rest of the system that reflects the behavior expected by $C$ from its environment in order to operate correctly. The goal of the local analyses is then to establish that every assumption made is also "guaranteed" - hence Assume-Guarantee.

Since its inception $[18,16]$, the AG paradigm has been explored in several directions. However, a major challenge in automating AG reasoning is constructing appropriate assumptions. For realistic systems, such assumptions are often complicated, and, therefore, constructing them manually is impractical. In this context, Cobleigh et al. [9] proposed the use of learning to automatically construct appropriate assumptions to verify a system composed of finite automata against a finite automaton specification (i.e., to verify safety properties). They used the following sound and complete AG proof rule:

$$
\frac{M_{1} \| A \sqsubseteq S \quad M_{2} \sqsubseteq A}{M_{1} \| M_{2} \sqsubseteq S}
$$

where $M_{1}, M_{2}, A$ and $S$ are finite automata, $\|$ is a parallel composition, and $\sqsubseteq$ denotes language containment. The essential idea is to use the $\mathbf{L}^{*}$ algorithm [2] to learn an assumption $A$ that satisfies the premises of the rule, and implement the minimally adequate teacher required by $\mathbf{L}^{*}$ via model-checking.

The learning-based automated AG paradigm has been extended in several directions [6, 1, 21]. However, the question of whether this paradigm is applicable to verifying $\omega$-regular properties (i.e., liveness and safety) of reactive systems is open. In this paper, we answer this question in the affirmative. An automated AG framework requires: (i) an algorithm that uses queries and counterexamples to learn an appropriate assumption, and (ii) a set of sound and complete AG rules. Recently, a learning algorithm for $\omega$-regular languages has been proposed by Farzan et al. [10]. However, to our knowledge, the AG proof rules have not been extended to $\omega$-regular properties. This is the problem we address in this paper.

First, we study the applicability of non-circular (AG-NC) and circular (AG-C) AG proof rules in the context of systems with infinite behaviors. We assume that processes synchronize on shared events and proceeding asynchronously otherwise, i.e., as in CSP [15]. We prove that, in this context, AG-NC is sound but incomplete when restricted to languages with strictly infinite behaviors (e.g., $\omega$-regular). This is surprising and interesting. In contrast, we show that AG-C is both sound and complete for $\omega$-regular languages. Second, we extend our AG proof rules to systems and specifications expressible in $\infty$-regular 
languages (i.e., unions of regular and $\omega$-regular languages). We show that both AG-C and AG-NC are sound and complete in this case. To the best of our knowledge, these soundness and completeness results are new. We develop two learning algorithms for $\infty$-regular languages - one using a learning algorithm for $\omega$-regular languages (see Theorem 8(a)) with an augmented alphabet, and another combining a learning algorithm for $\omega$-regular languages with $\mathbf{L}^{*}$ (see Theorem 8(b)) without alphabet augmentation. Finally, we present a very general formalization, called LAG, of the learning based automated AG paradigm. We show how existing approaches for automated AG reasoning are special instances of LAG. Furthermore, we show how to instantiate LAG to develop automated AG algorithms for $\infty$-regular, and $\omega$-regular, languages using both AG-NC and AG-C as proof rules.

The rest of the paper is structured as follows. We present the necessary background in Section 2. In Section 3, we review our model of concurrency. In Section 4, we study the soundness and completeness of AG rules, and present our LAG framework in Section 5. We conclude the paper with an overview of related work in Section 6.

\section{Preliminaries}

We write $\Sigma^{*}$ and $\Sigma^{\omega}$ for the set of all finite and infinite words over $\Sigma$, respectively, and write $\Sigma^{\infty}$ for $\Sigma^{*} \cup \Sigma^{\omega}$. We use the standard notation of regular expressions: $\lambda$ for empty word, $a \cdot b$ for concatenation, $a^{*}, a^{+}$, and $a^{\omega}$ for finite, finite and non-empty, and infinite repetition of $a$, respectively. When $a \in \Sigma^{\omega}$, we define $a \cdot b=a$. These operations are extended to sets in the usual way, e.g., $X \cdot Y=\{x \cdot y \mid x \in X \wedge y \in Y\}$.

Language. A language is a pair $(L, \Sigma)$ such that $\Sigma$ is an alphabet and $L \subseteq \Sigma^{\infty}$. The alphabet is an integral part of a language. In particular, $(\{a\},\{a\})$ and $(\{a\},\{a, b\})$ are different languages. However, for simplicity, we often refer to a language as $L$ and mention $\Sigma$ separately. For instance, we write "language $L$ over alphabet $\Sigma$ " to mean the language $(L, \Sigma)$, and $\Sigma(L)$ to mean the alphabet of $L$. Union and intersection are defined as usual, but only for languages over the same alphabet. The complement of $L$, denoted $\bar{L}$, is defined as: $\bar{L}=\Sigma(L)^{\infty} \backslash L$. A finitary language ( $\Sigma^{*}$-language) is a subset of $\Sigma^{*}$. An infinitary language ( $\Sigma^{\omega}$-language) is a subset of $\Sigma^{\omega}$. For $L \subseteq \Sigma^{\infty}$, we write $*(L)$ for the finitary language $L \cap \Sigma^{*}$ and $\omega(L)$ for the infinitary language $L \cap \Sigma^{\omega}$. Note that $\Sigma(\bar{L})=\Sigma(*(L))=\Sigma(\omega(L))=\Sigma(L)$.

Transition Systems. A labeled transition system (LTS) is a 4-tuple $M=(S, \Sigma$, Init, $\delta$ ), where $S$ is a finite set of states, $\Sigma$ is an alphabet, Init $\subseteq S$ is the set of initial states, and $\delta \subseteq S \times \Sigma \times S$ is a transition relation. We write $s \stackrel{\alpha}{\longrightarrow} s^{\prime}$ for $\left(s, \alpha, s^{\prime}\right) \in \delta$, and $\Sigma(M)$ for $\Sigma$. $M$ is deterministic if $\mid$ Init $\mid \leq 1$, and $\forall s \in S . \forall \alpha \in \Sigma .\left|\left\{s^{\prime} \mid s \stackrel{\alpha}{\longrightarrow} s^{\prime}\right\}\right| \leq 1$. A run $r$ over a word $w=\alpha_{0}, \alpha_{1}, \ldots, \in \Sigma(M)^{\infty}$ is a sequence of states $s_{0}, s_{1}, \ldots$, such that $\forall i \geq 0 . s_{i} \stackrel{\alpha_{i}}{\longrightarrow} s_{i+1}$. We write First $(r), \operatorname{Last}(r)$, and $\operatorname{Inf}(r)$ to denote the first state of $r$, the last state of $r$ (assuming $r \in S^{*}$ ), and states that occur infinitely often in $r$ (assuming $r \in S^{\omega}$ ), respectively. We write $\operatorname{Run}(w, M)$ for the set of runs of $w$ on $M$.

Automata. A Finite Automaton (FA) is a 5-tuple $A=(S, \Sigma$, Init $, \delta, F)$, where $(S, \Sigma$, Init, $\delta)$ is an LTS and $F \subseteq S$ is a set of accepting states. The language accepted by $A, \mathscr{L}(A)$, is the set of all words $w \in \Sigma^{*}$ s.t. there exists a run $r$ of $w$ on $A$, with First $(r) \in \operatorname{Init} \wedge \operatorname{Last}(r) \in F$. A BüchiAutomaton (BA) is a 5-tuple $B=(S, \Sigma$, Init $, \delta, F)$, where $(S, \Sigma$, Init,$\delta)$ is an LTS and $F \subseteq S$ is a set of accepting states. The language accepted by $B, \mathscr{L}(B)$, is the set of all words $w \in \Sigma^{\omega}$ s.t. there exists a run $r$ of $w$ on $A$ with First $(r) \in \operatorname{Init} \wedge \operatorname{Inf}(r) \cap F \neq \emptyset$. A BA or FA is deterministic if its underlying LTS is deterministic.

Regularity. A language is regular ( $\omega$-regular) iff it is accepted by a FA (BA). A language $L \subseteq \Sigma^{\infty}$ is $\infty$-regular iff $*(L)$ is regular and $\omega(L)$ is $\omega$-regular. Deterministic FA (DFA) and non-deterministic FA (NFA) are equally expressive. Deterministic BA are strictly less expressive than non-deterministic BA.

Learning. A learning algorithm for a regular language is any algorithm that learns an unknown, but fixed, language $U$ over a known alphabet $\Sigma$. Such an algorithm is called active if it works by querying a Minimally Adequate Teacher (MAT). The MAT can answer "Yes/No" to two types of queries about $U$ : 
Membership Query Given a word $w$, is $w \in U$ ?

Candidate Query Given an automaton $B$, is $\mathscr{L}(B)=U$ ? If the answer is "No", the MAT returns a counterexample $(C E)$, which is a word such that $C E \in \mathscr{L}(B) \ominus U$, where $X \ominus Y=(X \backslash Y) \cup(Y \backslash X)$.

An active learning algorithm begins by asking membership queries of the MAT until it constructs a candidate, with which it make a candidate query. If the candidate query is successful, the algorithm terminates; otherwise it uses the $C E$ returned by the MAT to construct additional membership queries. The family of active learning algorithms was originated by Angluin via $\mathbf{L}^{*}$ [2] for learning a minimal DFA that accepts an unknown regular language. $\mathbf{L}^{*}$ was further optimized by Rivest and Schapire [20].

The problem of learning a minimal automaton which accept an unknown $\omega$-regular language is still open. It is known [17] that for any language $U$ one can learn in the limit an automaton that accepts $U$ via the identification by enumeration approach proposed by Gold [12]. However, the automaton learned via enumeration may, in the worst case, be exponentially larger than the minimal automaton accepting $U$. Furthermore, there may be multiple minimal automata [17] accepting $U$. Maler et al. [17] have shown that $\mathbf{L}^{*}$ can be extended to learn a minimal (Müller) automaton for a fragment of $\omega$-regular languages.

Farzan et al. [10] show how to learn a Büchi automaton for an $\omega$-regular language $U$. Specifically, they use $\mathbf{L}^{*}$ to learn the language $U_{\$}=\left\{u \$ v \mid u \cdot v^{\omega} \in U\right\}$, where $\$$ is a fresh letter not in the alphabet of $U$. The language $U_{\$}$ was shown to be regular by Calbrix et al. [4]. In the sequel, we refer to this algorithm as $\mathbf{L}^{\$}$. The complexity of $\mathbf{L}^{\$}$ is exponential in the minimal BA for $U$. Our LAG framework can use any active algorithm for learning $\omega$-regular languages. In particular, $\mathbf{L}^{\$}$ is an existing candidate.

\section{Model of Concurrency}

Let $w$ be a word and $\Sigma$ an arbitrary alphabet. We write $w\rfloor \Sigma$ for the projection of $w$ onto $\Sigma$ defined recursively as follows (recall that $\lambda$ denotes the empty word):

$$
\lambda\rfloor \Sigma=\lambda \quad(a \cdot u)\rfloor \Sigma= \begin{cases}a \cdot(u\rfloor \Sigma) & \text { if } a \in \Sigma \\ u\rfloor \Sigma & \text { otherwise }\end{cases}
$$

Clearly, both $\Sigma^{*}$ and $\Sigma^{\infty}$ are closed under projection, but $\Sigma^{\omega}$ is not. For example, $\left.\left(a^{*} \cdot b^{\omega}\right\rfloor\{a\}\right)=a^{*}$, and $a^{*}$ consists only of finite words. Projection preservers regularity. If $L$ is a regular ( $\infty$-regular) language and $\Sigma$ is any alphabet, then $L\rfloor \Sigma$ is also regular ( $\infty$-regular).

A process is modeled by a language of all of its behaviors (or computations). Parallel composition ( ||$)$ of two processes/languages synchronizes on common actions while executing local actions asynchronously. For languages $\left(L_{1}, \Sigma_{1}\right)$ and $\left(L_{2}, \Sigma_{2}\right), L_{1} \| L_{2}$ is the language over $\Sigma_{1} \cup \Sigma_{2}$ defined as follows:

$$
\left.\left.L_{1} \| L_{2}=\left\{w \in\left(\Sigma_{1} \cup \Sigma_{2}\right)^{\infty} \mid w\right\rfloor \Sigma_{1} \in L_{1} \wedge w\right\rfloor \Sigma_{2} \in L_{2}\right\} \quad \text { (def. of } \| \text { ) }
$$

Intuitively, $L_{1}|| L_{2}$ consists of all permutations of words from $L_{1}$ and $L_{2}$ that have a common synchronization sequence. For example, $\left(b^{*} \cdot a \cdot b^{*}\right) \|\left(c^{*} \cdot a \cdot c^{*}\right)$ is $(b+c)^{*} \cdot a \cdot(b+c)^{*}$. Note that when $L_{1}$ and $L_{2}$ share an alphabet, the composition is their intersection; when their alphabets are disjoint, the composition is their language shuffle. The set of $\Sigma^{*}, \Sigma^{\omega}$, and $\Sigma^{\infty}$ languages are all closed under parallel composition.

Theorem 1. The || operator is associative, commutative, distributive over union and intersection. It is also monotone, i.e., for any two languages $L_{1}, L_{2}$, and $L_{3}: L_{2} \subseteq L_{3} \Rightarrow\left(L_{1}|| L_{2}\right) \subseteq\left(L_{1} \| L_{3}\right)$.

Let $L_{1}$ and $L_{2}$ be two languages such that $\Sigma\left(L_{1}\right) \supseteq \Sigma\left(L_{2}\right)$. We say that $L_{1}$ is subsumed by $L_{2}$, written $L_{1} \preccurlyeq L_{2}$, if $\left.L_{1}\right\rfloor \Sigma\left(L_{2}\right) \subseteq L_{2}$. Let $L_{S}$ be the language of a specification $S$, and $L_{M}$ be the language of a system $M$. Then, $M$ satisfies $S$, written $M \models S$, iff $L_{M} \preccurlyeq L_{S}$. 
Automated Assume-Guarantee Reasoning for Omega-Regular Systems and Specifications

\section{Proof Rules for Assume-Guarantee Reasoning}

In this section, we study the applicability of a non-circular and a circular AG rule to proving properties of processes with infinite behaviors (e.g., reactive systems that neither terminate nor deadlock). These rules were shown to be sound and complete for systems with finite (i.e., in $\Sigma^{*}$ ) behaviors by Barringer et al. [3]. In Section 4.1, we show that the non-circular AG rule is sound for both $\Sigma^{\infty}$ and $\Sigma^{\omega}$ behaviors. However, it is complete only when the assumptions are allowed to combine both finite and infinite behaviors (i.e., in $\left.\Sigma^{\infty}\right)$. In Section 4.2, we show that the circular AG rule is sound and complete for $\Sigma^{\omega}$ and $\Sigma^{\infty}$ behaviors.

\subsection{Non-Circular Assume-Guarantee Rule}

The non-circular AG proof rule (AG-NC for short) is stated as follows:

$$
\frac{\left(L_{1} \| L_{A}\right) \preccurlyeq L_{S} \quad L_{2} \preccurlyeq L_{A}}{\left(L_{1} \| L_{2}\right) \preccurlyeq L_{S}}
$$

where $L_{1}, L_{2}, L_{S}$, and $L_{A}$ are languages with the alphabets $\Sigma_{1}, \Sigma_{2}, \Sigma_{S}, \Sigma_{A}$, respectively, $\Sigma_{S} \subseteq\left(\Sigma_{1} \cup \Sigma_{2}\right)$, and $\Sigma_{A}=\left(\Sigma_{1} \cup \Sigma_{S}\right) \cap \Sigma_{2}$. AG-NC is known to be sound and complete for $\Sigma^{*}$-languages. Intuitively, it says that if there exists an assumption $L_{A}$ such that: (a) $L_{1}$ composed with $L_{A}$ is contained in $L_{S}$, and (b) $L_{2}$ is contained in $L_{A}$, then the composition of $L_{1}$ with $L_{2}$ is contained in $L_{S}$ as well. Note that the alphabet $\Sigma_{A}$ is the smallest alphabet containing: (a) actions at the interface between $L_{1}$ and $L_{2}$, i.e., actions common to the alphabets of $L_{1}$ and $L_{2}$, and (b) external actions of $L_{2}$, i.e., actions common to the alphabets of $L_{2}$ and $L_{S}$. Any smaller alphabet makes the rule trivially incomplete; any larger alphabet exposes internal (i.e., non-external) actions of $L_{2}$. It is not surprising that AG-NC remains sound even when applied to languages with infinite words. However, AG-NC is incomplete when $L_{A}$ is restricted to $\Sigma^{\omega}$-languages:

Theorem 2. There exists $L_{1}, L_{2}, L_{S} \subseteq \Sigma^{\omega}$ such that $\left(L_{1} \| L_{2}\right) \preccurlyeq L_{S}$, but there does not exists an assumption $L_{A} \subseteq \Sigma^{\omega}$ that satisfies all of the premises of AG-NC.

Proof. By example. Let $L_{1}, L_{2}, L_{S}$, and their alphabets be defined as follows:

$$
\Sigma_{1}=\{a, b\} \quad \Sigma_{2}=\{a, c\} \quad \Sigma_{S}=\{a, b\} \quad L_{1}=(a+b)^{\omega} \quad L_{2}=a^{*} c^{\omega} \quad L_{S}=(a+b)^{*} b^{\omega}
$$

The conclusion of AG-NC rule is satisfied since $\left.\left(L_{1}|| L_{2}\right)\right\rfloor \Sigma_{S}=(a+b)^{*} b^{\omega}=L_{S}$. The alphabet $\Sigma_{A}$ of $L_{A}$ is $\left(\Sigma_{1} \cup \Sigma_{S}\right) \cap \Sigma_{2}=\{a\}$. Since $L_{A} \subseteq \Sigma_{A}^{\omega}$, it can only be $a^{\omega}$ or $\emptyset$. The only way to satisfy the first premise of AG-NC is to let $L_{A}=\emptyset$, but this is too strong to satisfy the second premise.

Note that the proof of Theorem 2 shows that AG-NC is incomplete even for $\infty$-regular languages.

Remark 1. One may conjecture that the AG-NC rule becomes complete for $\Sigma^{\omega}$ if subsumption is redefined to only consider infinite words. That is, by redefining subsumption as: $L_{1} \preccurlyeq L_{2} \Leftrightarrow \omega\left(L_{1} \backslash \Sigma\left(L_{2}\right)\right) \subseteq$ $L_{2}$. However, under this interpretation, AG-NC is no longer sound. For example, let the languages $L_{1}$, $L_{2}, L_{S}$, and their alphabets be defined as follows:

$$
\Sigma_{1}=\{a, b\} \quad \Sigma_{2}=\{a, c\} \quad \Sigma_{S}=\{a, b\} \quad L_{1}=(a+b)^{\omega} \quad L_{2}=a^{*} c^{\omega} \quad L_{S}=b^{\omega}
$$

Then, the conclusion of AG-NC does not hold: $\left.\omega\left(\left(L_{1}|| L_{2}\right)\right\rfloor \Sigma_{S}\right)=(a+b)^{*} b^{\omega} \nsubseteq b^{\omega}$. But $L_{A}=\emptyset$ satisfies both premises: $\left(L_{1}|| L_{A}\right)=b^{\omega}$, and $\left.\omega\left(L_{2}\right\rfloor\{a\}\right)=L_{A}$.

Remark 2. AG-NC is complete if the alphabet $\Sigma_{A}$ is redefined to be $\Sigma_{1} \cup \Sigma_{2}$. However, in this case the rule is no longer "compositional" since the assumption $L_{A}$ can be as expressive as the component $L_{2}$.

Intuitively, AG-NC is incomplete for $\Sigma^{\omega}$ because $\Sigma^{\omega}$ is not closed under projection. However, we show that the rule is complete for $\Sigma^{\infty}$ - the smallest projection-closed extension of $\Sigma^{\omega}$. We first show that for any languages $L_{1}$ and $L_{S}$, there always exists a unique weakest assumption $L_{A}$, such that $L_{1} \| L_{A} \preccurlyeq L_{S}$. 
Theorem 3. Let $L_{1}$ and $L_{S}$ be two languages, and $\Sigma_{A}$ be any alphabet s.t. $\Sigma\left(L_{1}\right) \cup \Sigma_{A}=\Sigma\left(L_{1}\right) \cup \Sigma\left(L_{S}\right)$. Then, $L_{A}=\left\{w \in \Sigma_{A}^{\infty} \mid\left(L_{1}||\{w\}\right) \preccurlyeq L_{S}\right\}$ satisfies $L_{1} \| L_{A} \preccurlyeq L_{S}$, and is the weakest such assumption.

Proof. Let us write $\Sigma_{1}, \Sigma_{S}$ and $\Sigma_{1 S}$ to mean $\Sigma\left(L_{1}\right), \Sigma\left(L_{S}\right)$ and $\Sigma\left(L_{1}\right) \cup \Sigma\left(L_{S}\right)$ respectively. To show that $L_{A}$ is a valid assumption, pick any $w \in L_{1} \| L_{A}$. Then $w \backslash \Sigma_{A} \in L_{A}$. This implies that $\left.w\right\rfloor \Sigma_{S} \in$ $\left.\left.\left(L_{1} \|\{w\rfloor \Sigma_{A}\right\}\right)\right\rfloor \Sigma_{S} \subseteq L_{S}$. Since $w$ is any word in $L_{1} \| L_{A}$, we have $L_{1} \| L_{A} \preccurlyeq L_{S}$. To show that $L_{A}$ is the weakest assumption, let $L_{A}^{\prime} \subseteq \Sigma_{A}^{\infty}$ be any language such that $L_{1} \| L_{A}^{\prime} \preccurlyeq L_{S}$ and let $w$ be any word in $L_{A}^{\prime}$. Then, $\left(L_{1} \|\{w\}\right) \subseteq\left(L_{1} \| L_{A}^{\prime}\right) \preccurlyeq L_{S}$. But this implies that $w \in L_{A}$, and, therefore, $L_{A}^{\prime} \subseteq L_{A}$.

Note that $\Sigma_{A}^{\infty}$ subsumes both finite $\left(\Sigma_{A}^{*}\right)$ and infinite $\left(\Sigma_{A}^{\omega}\right)$ words. Thus, if $L_{A}$ is a $\Sigma_{A}^{\infty}$ weakest assumption, then $*\left(L_{A}\right)$ and $\omega\left(L_{A}\right)$ are the weakest $\Sigma_{A}^{*}$ and $\Sigma_{A}^{\omega}$ assumptions, respectively.

Theorem 4. Let $L_{1}, L_{2}, L_{S}$, and $L_{A}$ be in $\Sigma^{\infty}$. Then, the AG-NC rule is sound and complete.

Proof. The proof of soundness is trivial and is omitted. For the proof of completeness we only show the key step. Assume that $L_{1} \| L_{2} \preccurlyeq L_{S}$, and let $L_{A}$ be the weakest assumption such that $L_{1} \| L_{A} \preccurlyeq L_{S}$. By Theorem 3, $L_{A}$ is well-defined and satisfies the first premise of AG-NC. The second premise holds because $L_{2} \downarrow \Sigma_{A} \subseteq \Sigma_{A}^{\infty}$, and $L_{A}$ is the weakest $\Sigma_{A}^{\infty}$ assumption (see Theorem 3).

Theorem 4 implies that AG-NC is sound for any fragment of $\Sigma^{\infty}$. Of course, this is not true for completeness of the rule. For practical purposes, we would like to know that the rule remains complete when its languages are restricted to the regular subset. We show that this is so by showing that under the assumption that $L_{1}$ and $L_{S}$ are regular, the weakest assumption is regular as well.

Theorem 5. Let $L_{1}$ and $L_{S}$ be two languages, and $\Sigma_{A}$ be any alphabet such that $\Sigma\left(L_{1}\right) \cup \Sigma_{A}=\Sigma\left(L_{1}\right) \cup$ $\Sigma\left(L_{S}\right)$. Then, $L_{A} \subseteq \Sigma_{A}^{\infty}$ is the weakest assumption such that $L_{1} \| L_{A} \preccurlyeq L_{S}$ iff $L_{A}=\overline{\left.\left(L_{1} \| \overline{L_{S}}\right)\right\rfloor \Sigma_{A}}$.

Proof. Let us write $\Sigma_{1}, \Sigma_{S}$ and $\Sigma_{1 S}$ to mean $\Sigma\left(L_{1}\right), \Sigma\left(L_{S}\right)$ and $\Sigma\left(L_{1}\right) \cup \Sigma\left(L_{S}\right)$, respectively. For any $w \in \Sigma_{A}^{\infty}$ :

$$
\begin{array}{ll} 
& w \in \overline{\left.\left(L_{1} \| \overline{L_{S}}\right)\right\rfloor \Sigma_{A}} \text { iff } \forall w^{\prime} \in \Sigma_{1 S}^{\infty} \cdot\left\{w^{\prime}\right\} \preccurlyeq\{w\} \Longrightarrow w^{\prime} \notin\left(L_{1} \| \overline{L_{S}}\right) \\
\text { iff } \quad \forall w^{\prime} \in \Sigma_{1 S}^{\infty} \cdot\left\{w^{\prime}\right\} \preccurlyeq\{w\} \Longrightarrow\left(\left\{w^{\prime}\right\} \preccurlyeq L_{1} \vee\left\{w^{\prime}\right\} \preccurlyeq L_{S}\right) \\
\text { iff } \forall w^{\prime} \in \Sigma_{1 S}^{\infty} \cdot\left(\left\{w^{\prime}\right\} \preccurlyeq\{w\} \wedge\left\{w^{\prime}\right\} \preccurlyeq L_{1}\right) \Longrightarrow\left\{w^{\prime}\right\} \preccurlyeq L_{S} \\
\text { iff } \forall w^{\prime} \in \Sigma_{1 S}^{\infty} \cdot\left(\left\{w^{\prime}\right\} \preccurlyeq\left(L_{1} \|\{w\}\right)\right) \Longrightarrow\left\{w^{\prime}\right\} \preccurlyeq L_{S} \text { iff } L_{1} \|\{w\} \preccurlyeq L_{S}
\end{array}
$$

Together with Theorem 3, this completes the proof.

Theorem 5 implies AG-NC is complete for any class of languages closed under complementation and projection, e.g., regular and $\infty$-regular languages. In addition, Theorem 5 implies that learningbased automated AG reasoning is effective for any class of languages whose weakest assumptions fall in a "learnable" fragment. In particular, this holds for regular, $\omega$-regular and $\infty$-regular languages.

\subsection{Circular Assume-Guarantee Rule}

The Circular Assume-Guarantee proof rule (AG-C for short) is stated as follows:

$$
\frac{\left(L_{1} \| L_{A 1}\right) \preccurlyeq L_{S} \quad\left(L_{2} \| L_{A 2}\right) \preccurlyeq L_{S} \quad\left(\overline{L_{A 1}} \| \overline{L_{A 2}}\right) \preccurlyeq L_{S}}{\left(L_{1} \| L_{2}\right) \preccurlyeq L_{S}}
$$

where $L_{1}, L_{2}$, and $L_{S}$ are languages over alphabets $\Sigma_{1}, \Sigma_{2}, \Sigma_{S}$, respectively; $\Sigma_{S} \subseteq \Sigma_{1} \cup \Sigma_{2}$, and $L_{A 1}$ and $L_{A 2}$ share a common alphabet $\Sigma_{A}=\left(\Sigma_{1} \cap \Sigma_{2}\right) \cup \Sigma_{S}$. AG-C is known to be sound and complete for $\Sigma^{*}$ languages. Note that in comparison with AG-NC, there are two assumptions $L_{A 1}$ and $L_{A 2}$ over a larger alphabet $\Sigma_{A}$. Informally, the rule is sound for the following reason. Let $w$ be a word in $L_{1}|| L_{2}$, and $u=w\rfloor \Sigma_{A}$. Then $u \in L_{A 1}$, or $u \in L_{A 2}$, or $u \in \overline{L_{A 1} \cup L_{A 2}}=\left(\overline{L_{A 1}} \| \overline{L_{A 2}}\right)$. If $u \in L_{A 1}$ then the first premise implies that $\{w\} \preccurlyeq L_{1} \|\{u\} \preccurlyeq L_{S}$; if $u \in L_{A 2}$ then the second premise implies that $\{w\} \preccurlyeq L_{2} \|\{u\} \preccurlyeq L_{S}$; otherwise, the third premise implies that $\{w\} \preccurlyeq\{u\} \preccurlyeq L_{S}$. 
Automated Assume-Guarantee Reasoning for Omega-Regular Systems and Specifications

Remark 3. Note that the assumption alphabet for AG-C is larger than AG-NC. In fact, using $\Sigma_{A 1}=$ $\left(\Sigma_{1} \cup \Sigma_{S}\right) \cap \Sigma_{2}$ and $\Sigma_{A 2}=\left(\Sigma_{2} \cup \Sigma_{S}\right) \cap \Sigma_{1}$ makes AG-C incomplete. Indeed, let $L_{1}=\{a a\}$ with $\Sigma_{1}=\{a\}$, $L_{2}=\{b b\}$ with $\Sigma_{2}=\{b\}$ and $\overline{L_{S}}=\{a a b, a b b, a b\}$. Note that $L_{1} \| L_{2} \preccurlyeq L_{S}$. We show that no $L_{A_{1}}$ and $L_{A_{2}}$ can satisfy the three premises of AG-C. Premise $1 \Rightarrow b \notin L_{A_{1}} \Rightarrow b \in \overline{L_{A_{1}}}$. Similarly, premise 2 $\Rightarrow a \notin L_{A_{2}} \Rightarrow a \in \overline{L_{A_{2}}}$. But then $a b \in \overline{L_{A 1}}|| \overline{L_{A_{2}}}$, violating premise 3 .

In this section, we show that AG-C is sound and complete for both $\Sigma^{\omega}$ and $\Sigma^{\infty}$ languages. First, we illustrate an application of the rule to the example from the proof of Theorem 2. Let $L_{1}, L_{2}$, and $L_{S}$ be $\Sigma^{\omega}$ languages as defined in the proof of Theorem 2. In this case, the alphabet $\Sigma_{A}$ is $\{a, b\}$. Letting $L_{A 1}=(a+b)^{*} b^{\omega}$, and $L_{A 2}=(a+b)^{\omega}$ satisfies all three premises of the rule.

Theorem 6. Let $L_{1}, L_{2}, L_{S}, L_{A 1}$, and $L_{A 2}$ be in $\Sigma^{\omega}$ or $\Sigma^{\infty}$. Then, the AG-C rule is sound and complete.

Proof. The proof of soundness is sketched in the above discussion. For the proof of completeness we only show the key steps. Assume that $L_{1}|| L_{2} \preccurlyeq L_{S}$. Let $L_{A 1}$ and $L_{A 2}$ be the weakest assumptions such that $L_{1}|| L_{A 1} \preccurlyeq L_{S}$, and $L_{2}|| L_{A 2} \preccurlyeq L_{S}$, respectively. By Theorem 3, both $L_{A 1}$ and $L_{A 2}$ are well-defined and satisfy the first and the second premises of AG-C, respectively. We prove the third premise by contradiction. Since $L_{A 1}$ and $L_{A 2}$ have the same alphabet, $\left(\overline{L_{A 1}}|| \overline{L_{A 2}}\right)=\left(\overline{L_{A 1}} \cap \overline{L_{A 2}}\right)$. Assume that $\left(\overline{L_{A 1}} \cap\right.$ $\left.\overline{L_{A 2}}\right) \npreceq L_{S}$. Then, there exists a word $w \in\left(\overline{L_{A 1}}|| \overline{L_{A 2}}\right)$ such that $w \notin L_{A 1}$, and $w \notin L_{A 2}$, and $\left.w\right\rfloor \Sigma_{S} \notin L_{S}$. By the definition of weakest assumption (see Theorem 3), $L_{1} \|\{w\} \npreceq L_{S}$ and $L_{2} \|\{w\} \npreceq L_{S}$. Pick any $w_{1} \in L_{1} \|\{w\}$ and $w_{2} \in L_{2} \|\{w\}$. Let $w_{1}^{\prime}=w_{1} \backslash \Sigma_{1}$ and $\left.w_{2}^{\prime}=w_{2}\right\rfloor \Sigma_{2}$. We know that $\left\{w_{1}^{\prime}\right\}\left\|\left\{w_{2}^{\prime}\right\} \subseteq L_{1}\right\| L_{2}$. Also, $\left.w \in\left(\left\{w_{1}^{\prime}\right\} \|\left\{w_{2}^{\prime}\right\}\right)\right\rfloor \Sigma_{A}$. Now since $\left\{w_{1}^{\prime}\right\}||\left\{w_{2}^{\prime}\right\} \subseteq L_{1} \| L_{2}$, we have $w \in\left(L_{1} \| L_{2}\right) \backslash \Sigma_{A}$. Since $\Sigma_{S} \subseteq \Sigma_{A}$, $\left.w\rfloor \Sigma_{S} \in\left(L_{1}|| L_{2}\right)\right\rfloor \Sigma_{S}$. But $\left.w\right\rfloor \Sigma_{S} \notin L_{S}$, which contradicts $L_{1}|| L_{2} \preccurlyeq L_{S}$.

The completeness part of the proof of Theorem 6 is based on the existence of the weakest assumption. We already know from Theorem 5, that the weakest assumption is ( $\infty-, \omega-)$ regular if $L_{1}, L_{2}$, and $L_{S}$ are $\left(\infty_{-}, \omega-\right)$ regular, respectively. Thus, AG-C is complete for $\left(\infty_{-}, \omega-\right)$ regular languages. Since AG-NC is incomplete for $\omega$-regular languages, a learning algorithm for $\omega$-regular languages (such as $\mathbf{L}^{\$}$ ) cannot be applied directly for AG reasoning for $\omega$-regular systems and specifications. In the next section, we overcome this challenge by developing automated AG algorithms for $\infty$-regular and $\omega$-regular languages.

\section{Automated Assume-Guarantee Reasoning}

In this section, we present our LAG framework, and its specific useful instances. LAG uses membership oracles, learners, and checkers, which we describe first.

Definition 1 (Membership Oracle and Learner). A membership oracle $Q$ for a language $U$ over alphabet $\Sigma$ is a procedure that takes as input a word $u \in \Sigma^{\infty}$ and returns 0 or 1 such that $Q(u)=1 \Longleftrightarrow u \in U$. We say that $Q=U$. The set of all membership oracles is denoted by Oracle. Let $\mathscr{A}$ be any set of automata. We write Learner $\mathscr{A}$ to denote the set of all learners of type $\mathscr{A}$. Formally, a learner of type $\mathscr{A}$ is a pair (Cand, LearnCE) such that: (i) Cand : Oracle $\mapsto \mathscr{A}$ is a procedure that takes a membership oracle as input and outputs a candidate $C \in \mathscr{A}$, and (ii) LearnCE : $\Sigma^{\infty} \mapsto$ Learner $_{\mathscr{A}}$ is a procedure that takes a counterexample as input and returns a new learner of type $\mathscr{A}$. For any learner $P=(\mathbf{C a n d}, \mathbf{L e a r n C E})$ we write P.Cand and P.LearnCE to mean Cand and LearnCE respectively.

Intuitively, a membership oracle is the fragment of a MAT that only answers membership queries, while a learner encapsulates an active learning algorithm that is able to construct candidates via membership queries, and learn from counterexamples of candidate queries.

Learning. Let $U$ be any unknown language, $Q$ be an oracle, and $P$ be a learner. We say that $(P, Q)$ learns $U$ if the following holds: if $Q \models U$, then there does not exist an infinite sequence of learners $P_{0}, P_{1}, \ldots$ and an infinite sequence of counterexamples $C E_{1}, C E_{2}, \ldots$ such that: (i) $P_{0}=P$, (ii) $P_{i}=P_{i-1}$.LearnCE $\left(C E_{i}\right)$ for $i>0$, and (iii) $C E_{i} \in \mathscr{L}\left(P_{i-1}\right.$. Cand $\left.(Q)\right) \ominus U$ for $i>0$. 


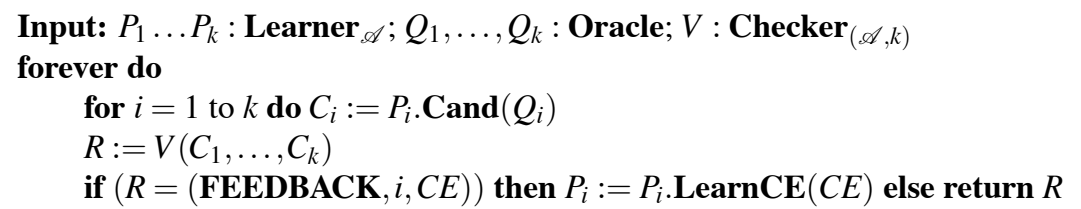

Figure 1: Algorithm for overall LAG procedure.

Definition 2 (Checker). Let $\mathscr{A}$ be a set of automata, and $k$ be an integer denoting the number of candidates. A checker of type $(\mathscr{A}, k)$ is a procedure that takes as input $k$ elements $A_{1}, \ldots, A_{k}$ of $\mathscr{A}$ and returns either (i) SUCCESS, or (ii) a pair (FAILURE, $C E$ ) such that $C E \in \Sigma^{\infty}$, or (iii) a triple $($ FEEDBACK, $i, C E)$ such that $1 \leq i \leq k$ and $C E \in \Sigma^{\infty}$. We write $\mathbf{C h e c k e r}_{(\mathscr{A}, k)}$ to mean the set of all checkers of type $(\mathscr{A}, k)$.

Intuitively, a checker generalizes the fragment of a MAT that responds to candidate queries by handling multiple (specifically, $k$ ) candidates. This generalization is important for circular proof rules. The checker has three possible outputs: (i) SUCCESS if the overall verification succeeds; (ii) (FAILURE, $C E$ ) where $C E$ is a real counterexample; (iii) (FEEDBACK, $i, C E$ ) where $C E$ is a counterexample for the $i$-th candidate.

\subsection{LAG Procedure}

Our overall LAG procedure is presented in Fig. 1. We write $X: T$ to mean that " $X$ is of type $T$ ". LAG accepts a set of $k$ membership oracles, $k$ learners, and a checker, and repeats the following steps:

1. Constructs candidate automata $C_{1}, \ldots, C_{k}$ using the learners and oracles.

2. Invokes the checker with the candidates constructed in Step 1 above.

3. If the checker returns SUCCESS or (FAILURE, $C E$ ), then exits with this result. Otherwise, updates the appropriate learner with the feedback and repeats from Step 1.

Theorem 7. $L A G$ terminates if there exists languages $U_{1}, \ldots, U_{k}$ such that: ( $\left.i\right) Q_{i}=U_{i}$ for $1 \leq i \leq k$, (ii) $\left(P_{i}, Q_{i}\right)$ learns $U_{i}$ for $1 \leq i \leq k$, and (iii) if $V\left(C_{1}, \ldots, C_{k}\right)=($ FEEDBACK, $i, C E)$, then $C E \in \mathscr{L}\left(C_{i}\right) \ominus U_{i}$.

Proof. By contradiction. If LAG does not terminate there exists some $P_{i}$ such that $P_{i}$. LearnCE is called infinitely often. This, together with assumptions (i) and (iii), contradicts (ii), i.e., $\left(P_{i}, Q_{i}\right)$ learns $U_{i}$.

\subsection{Oracle, Learner, and Checker Instantiations}

We now describe various implementations of oracles, learners and checkers. We start with the notion of an oracle for weakest assumptions.

Oracle for Weakest Assumption. Let $L_{1}, L_{S}$ be any languages and $\Sigma$ be any alphabet. We write $Q\left(L_{1}, L_{S}, \Sigma\right)$ to denote the oracle such that $Q\left(L_{1}, L_{S}, \Sigma\right) \models \overline{\left(L_{1} \| \overline{L_{S}}\right) \downarrow \Sigma} . Q\left(L_{1}, L_{S}, \Sigma\right)$ is typically implemented via model checking since, by Theorems 3 and $5, Q\left(L_{1}, L_{S}, \Sigma\right)(u)=1 \Longleftrightarrow u \in \Sigma^{\infty} \wedge L_{1} \|\{u\} \preccurlyeq L_{S}$.

Learner Instantiations. In general, a learner $P(\mathbf{L})$ is derived from an active learning algorithm $\mathbf{L}$ as follows: $P(\mathbf{L})=(\mathbf{C a n d}, \mathbf{L e a r n C E})$ s.t. $\mathbf{C a n d}=$ part of $\mathbf{L}$ that constructs a candidate using membership queries, and $\mathbf{L e a r n C E}=$ part of $\mathbf{L}$ that learns from a counterexample to a candidate query.

Non-circular Checker. Let $\mathscr{A}$ be a type of automata, and $L_{1}, L_{2}$ and $L_{S}$ be any languages. Then $V_{N C}\left(L_{1}, L_{2}, L_{S}\right)$ is the checker of type $(\mathscr{A}, 1)$ defined in Fig. 2. Note that $V_{N C}\left(L_{1}, L_{2}, L_{S}\right)$ is based on the AG-NC proof rule. The following proposition about $V_{N C}\left(L_{1}, L_{2}, L_{S}\right)$ will be used later.

Proposition 1. If $V_{N C}\left(L_{1}, L_{2}, L_{S}\right)(A)$ returns SUCCESS, then $L_{1} \| L_{2} \preccurlyeq L_{S}$. Otherwise, if $V_{N C}\left(L_{1}, L_{2}, L_{S}\right)(A)$ returns (FAILURE, $\left.C E\right)$, then $C E$ is a valid counterexample to $L_{1} \| L_{2} \preccurlyeq L_{S}$. Finally, if $V_{N C}\left(L_{1}, L_{2}, L_{S}\right)(A)$ returns (FEEDBACK, $\left.1, C E\right)$, then $C E \in \mathscr{L}(A) \ominus \overline{\left(L_{1} \| \overline{L_{S}}\right) \downarrow \Sigma}$. 
Automated Assume-Guarantee Reasoning for Omega-Regular Systems and Specifications

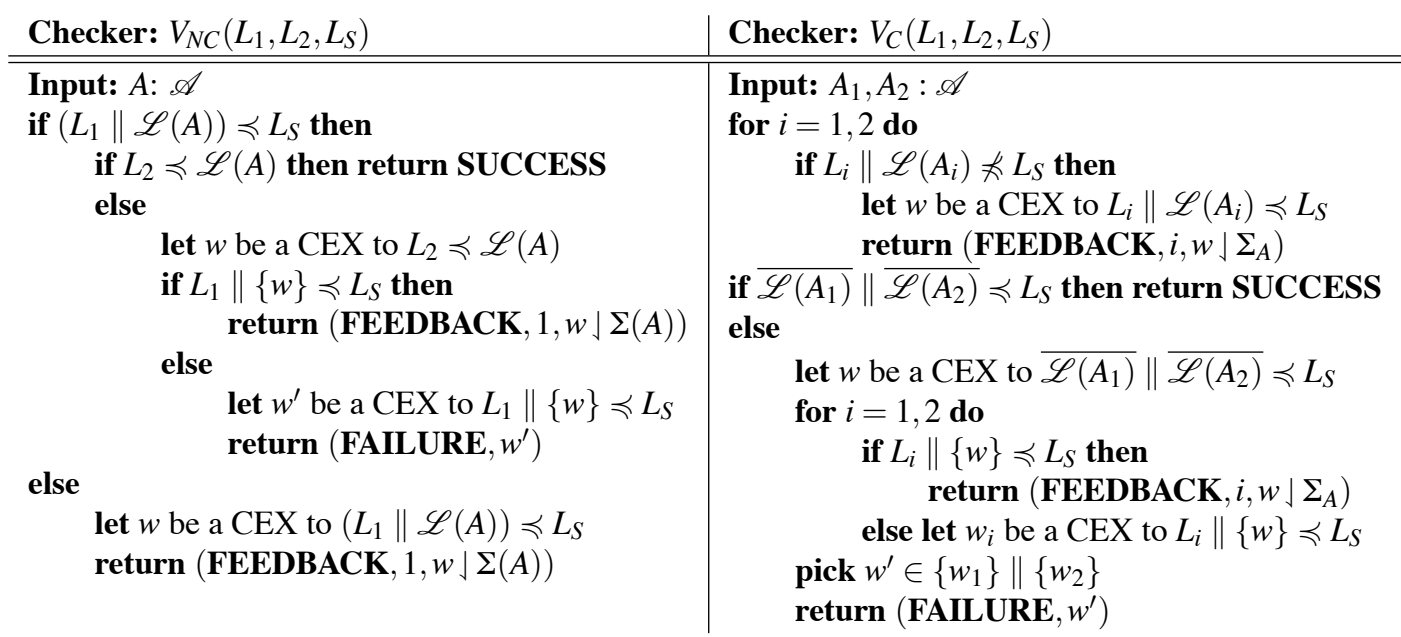

Figure 2: $V_{N C}-$ a checker based on AG-NC; $V_{C}$ - a checker based on AG-C.

Circular Checker. Let $\mathscr{A}$ be a type of automata, and $L_{1}, L_{2}$ and $L_{S}$ be any languages. Then $V_{C}\left(L_{1}, L_{2}, L_{S}\right)$ is the checker of type $(\mathscr{A}, 2)$ defined in Fig. 2. Note that $V_{C}\left(L_{1}, L_{2}, L_{S}\right)$ is based on the AG-C proof rule. The following proposition about $V_{C}\left(L_{1}, L_{2}, L_{S}\right)$ will be used later.

Proposition 2. If $V_{C}\left(L_{1}, L_{2}, L_{S}\right)\left(A_{1}, A_{2}\right)$ returns SUCCESS, then $L_{1} \| L_{2} \preccurlyeq L_{S}$. Otherwise, if $V_{C}\left(L_{1}, L_{2}, L_{S}\right)\left(A_{1}, A_{2}\right)$ returns (FAILURE, $\left.C E\right)$, then $C E$ is a valid counterexample to $L_{1} \| L_{2} \preccurlyeq L_{S}$. Finally, if $V_{C}\left(L_{1}, L_{2}, L_{S}\right)\left(A_{1}, A_{2}\right)$ returns $($ FEEDBACK, $i, C E)$, then $C E \in \mathscr{L}\left(A_{i}\right) \ominus \overline{\left(L_{i} \| \overline{L_{S}}\right) \downarrow \Sigma}$.

\subsection{LAG Instantiations}

In this section, we present several instantiations of LAG for checking $L_{1} \| L_{2} \preccurlyeq L_{S}$. Our approach extends to systems with finitely many components, as for example in $[9,3]$.

Existing Work as LAG Instances: Regular Trace Containment. Table 1 instantiates LAG for existing learning-based algorithms for AG reasoning. The first row corresponds to the work of Cobleigh et al. [9]; its termination and correctness follow from Theorem 7, Proposition 1, and the fact that $\left(P_{1}, Q_{1}\right)$ learns the language $\overline{\left(L_{1} \| \overline{L_{S}}\right) \downarrow \Sigma}$. The second row corresponds to Barringer et al. [3]; its termination and correctness follow from Theorem 7, Proposition 2, and the fact that $\left(P_{i}, Q_{i}\right)$ learns $\overline{\left(L_{i} \| \overline{L_{S}}\right) \downarrow \Sigma}$ for $i \in\{1,2\}$.

New Contribution: Learning Infinite Behavior. Let $\mathbf{L}^{\omega}$ be any active learning algorithm for $\omega$-regular languages (e.g., $\mathbf{L}^{\$}$ ). Since $\mathbf{A G - N C}$ is incomplete for $\omega$-regular languages, $\mathbf{L}^{\omega}$ is not applicable directly in this context. On the other hand, both AG-NC and AG-C are sound and complete for $\infty$-regular languages. Therefore, a learning algorithm for $\infty$-regular languages yields LAG instances for systems with infinite behavior. We now present two such algorithms. The first (see Theorem 8 (a)) uses $\mathbf{L}^{\omega}$ only, but augments the assumption alphabet. The second (see Theorem 8(b)) combines $\mathbf{L}^{\omega}$ and $\mathbf{L}^{*}$, but leaves the assumption alphabet unchanged. We present both schemes since neither is objectively superior.

Theorem 8. We can learn a $\infty$-regular language $U$ using a MAT for $U$ in two ways: (a) using only $\mathbf{L}^{\omega}$ but with alphabet augmentation, and (b) without alphabet augmentation, but using both $\mathbf{L}^{*}$ and $\mathbf{L}^{\omega}$.

Proof. Part(a): Let $\Sigma$ be the alphabet of $U$. We use $\mathbf{L}^{\omega}$ to learn an $\omega$-regular language $U^{\prime}$ over the alphabet $\Sigma^{\prime}=\Sigma \cup\{\tau\}$ such that $\left.U^{\prime}\right\rfloor \Sigma=U$, and $\tau \notin \Sigma$. Let $U^{\prime}=U \cdot \tau^{\omega}$. We assume that the MAT $X$ for $U$ accepts membership queries of the form $\left(M_{1}, M_{2}\right) \in \mathrm{DFA} \times \mathrm{BA}$, and returns "Yes" if $U=$ $\mathscr{L}\left(M_{1}\right) \cup \mathscr{L}\left(M_{2}\right)$, and a $C E$ otherwise. Then, a MAT for $U^{\prime}$ is implemented using $X$ as follows: (i) Membership: $u \in U^{\prime}$ iff $\left.u \in \Sigma^{\infty} \cdot \tau^{\omega} \wedge u\right\rfloor \Sigma \in U$, where $\left.u\right\rfloor \Sigma \in U$ is decided using $X$; (ii) Candidate 
Sagar Chaki and Arie Gurfinkel

\begin{tabular}{|c|c|c|c|c|c|}
\hline Conformance & Rule & $\mathscr{A}$ & Learner(s) & Oracle(s) & Checker \\
\hline $\begin{array}{c}\text { Regular Trace } \\
\text { Containment }\end{array}$ & $\begin{array}{c}\text { AG-NC } \\
{[9]}\end{array}$ & DFA & $P_{1}=P\left(\mathbf{L}^{*}\right)$ & $Q_{1}=Q\left(L_{1}, L_{S}, \Sigma_{N C}\right)$ & $V_{N C}\left(L_{1}, L_{2}, L_{S}\right)$ \\
\hline $\begin{array}{c}\text { Regular Trace } \\
\text { Containment }\end{array}$ & AG-C & DFA & $\begin{array}{c}P_{1}=P_{2}= \\
P\left(\mathbf{L}^{*}\right)\end{array}$ & $\begin{array}{c}Q_{1}=Q\left(L_{1}, L_{S}, \Sigma_{C}\right) \\
Q_{2}=Q\left(L_{2}, L_{S}, \Sigma_{C}\right)\end{array}$ & $V_{C}\left(L_{1}, L_{2}, L_{S}\right)$ \\
\hline $\begin{array}{c}\infty \text {-regular Trace } \\
\text { Containment }\end{array}$ & AG-NC & DFA $\times$ BA & $P_{1}=P(\mathbf{L})$ & $Q_{1}=Q\left(L_{1}, L_{S}, \Sigma_{N C}\right)$ & $V_{N C}\left(L_{1}, L_{2}, L_{S}\right)$ \\
\hline $\begin{array}{c}\infty \text {-regular Trace } \\
\text { Containment }\end{array}$ & AG-C & DFA $\times$ BA & $\begin{array}{c}P_{1}=P_{2}= \\
P(\mathbf{L})\end{array}$ & $\begin{array}{c}Q_{1}=Q\left(L_{1}, L_{S}, \Sigma_{C}\right) \\
Q_{2}=Q\left(L_{2}, L_{S}, \Sigma_{C}\right)\end{array}$ & $V_{C}\left(L_{1}, L_{2}, L_{S}\right)$ \\
\hline$\omega$-regular Trace & AG-NC & DFA $\times$ BA & $P_{1}=P(\mathbf{L})$ & $Q_{1}=Q\left(L_{1}, L_{S}, \Sigma_{N C}\right)$ & $V_{N C}\left(L_{1}, L_{2}, L_{S}\right)$ \\
Containment & & & & & \\
\hline$\omega$-regular Trace & AG-C & BA & $P_{1}=P_{2}=$ & $Q_{1}=Q\left(L_{1}, L_{S}, \Sigma_{C}\right)$ & $V_{C}\left(L_{1}, L_{2}, L_{S}\right)$ \\
Containment & & & $P\left(\mathbf{L}^{\omega}\right)$ & $Q_{2}=Q\left(L_{2}, L_{S}, \Sigma_{C}\right)$ & \\
\hline
\end{tabular}

Table 1: Existing learning-based AG algorithms as instances of LAG; $\Sigma_{N C}=\left(\Sigma\left(L_{1}\right) \cup \Sigma\left(L_{S}\right)\right) \cap \Sigma\left(L_{2}\right)$; $\Sigma_{C}=\left(\Sigma\left(L_{1}\right) \cap \Sigma\left(L_{2}\right)\right) \cup \Sigma\left(L_{S}\right) ; \mathbf{L}$ is a learning algorithm from Theorem 8 .

with $C^{\prime}$ : If $\mathscr{L}\left(C^{\prime}\right) \nsubseteq \Sigma^{\infty} \cdot \tau^{\omega}$, return $C E^{\prime} \in \mathscr{L}\left(C^{\prime}\right) \backslash \Sigma^{\infty} \cdot \tau^{\omega}$. Otherwise, make a candidate query to $X$ with $\left(M_{1}, M_{2}\right)$ such that $\mathscr{L}\left(M_{1}\right)=*\left(C^{\prime} \backslash \Sigma\right)$ and $\mathscr{L}\left(M_{2}\right)=\omega\left(C^{\prime} \backslash \Sigma\right)$, and turn any $C E$ to $C E^{\prime}=C E \cdot \tau^{\omega}$.

Part(b): We use $\mathbf{L}^{*}$ to learn $*(U)$ and $\mathbf{L}^{\omega}$ to learn $\omega(U)$. We assume that the MAT $X$ for $U$ accepts membership queries of the form $\left(M_{1}, M_{2}\right) \in \mathrm{DFA} \times \mathrm{BA}$, and returns "Yes" if $U=\mathscr{L}\left(M_{1}\right) \cup \mathscr{L}\left(M_{2}\right)$, and a $C E$ otherwise. We run $\mathbf{L}^{*}$ and $\mathbf{L}^{\omega}$ concurrently, and iterate the two next steps: (1) answer membership queries with $X$ until we get candidates $M_{1}$ and $M_{2}$ from $\mathbf{L}^{*}$ and $\mathbf{L}^{\omega}$ respectively; (2) make candidate query $\left(M_{1}, M_{2}\right)$ to $X$; return any finite (infinite) $C E$ back to $\mathbf{L}^{*}\left(\mathbf{L}^{\omega}\right)$; repeat from Step 1.

LAG instances for $\infty$-regular Trace Containment. Suppose that $L_{1}, L_{2}$ and $L_{S}$ are $\infty$-regular and we wish to verify $L_{1} \| L_{2} \preccurlyeq L_{S}$. The third row of Table 1 show how to instantiate LAG to solve this problem using AG-NC. This instance of LAG terminates with the correct result due to Theorem 7, Proposition 1, and the fact that $\left(P_{1}, Q_{1}\right)$ learns $\overline{\left(L_{1} \| \overline{L_{S}}\right) \downarrow \Sigma}$. The fourth row of Table 1 show how to instantiate LAG to solve this problem using AG-C. This instance of LAG terminates correctly due to Theorem 7, Proposition 2 , and because $\left(P_{i}, Q_{i}\right)$ learns $\overline{\left.\left(L_{i} \| \overline{L_{S}}\right)\right\rfloor \Sigma}$ for $i \in\{1,2\}$.

LAG instances for $\omega$-regular Trace Containment. Suppose that $L_{1}, L_{2}$ and $L_{S}$ are $\omega$-regular and we wish to check $L_{1} \| L_{2} \preccurlyeq L_{S}$. When using AG-NC, restricting assumptions to $\omega$-regular languages is incomplete (cf. Theorem 2). Hence, the situation is the same as for $\infty$-regular languages (cf. row 5 of Table 1). When using AG-C, restricting assumptions to be $\omega$-regular is complete (cf. Theorem 6). Hence, we use $\mathbf{L}^{\omega}$ without augmenting the assumption alphabet, as summarized in row 6 of Table 1 . This is a specific benefit of the restriction to $\omega$-regular languages. This instance terminates with the correct result due to Theorem 7, Proposition 2, and because $\left(P_{i}, Q_{i}\right)$ learns $\overline{\left.\left(L_{i} \| \overline{L_{S}}\right)\right\rfloor \Sigma}$ for $i \in\{1,2\}$.

\section{Related Work and Conclusion}

Automated AG reasoning with automata-based learning was pioneered by Cobleigh et al. [9] for checking safety properties of finite state systems. In this context, Barringer et al. [3] investigate the soundness and completeness of a number of decomposition proof rules, and Wang [23] proposed a framework for automatic derivation of sound decomposition rules. Here, we extend the AG reasoning paradigm to arbitrary $\omega$-regular properties (i.e., both safety and liveness) using both non-circular and circular rules.

The idea behind (particular instances of) Theorem 5 is used implicitly in almost all existing work on automated assume-guarantee reasoning $[9,6,7]$. However, we are not aware of an explicit closed-form treatment of the weakest assumption in a general setting such as ours.

The learning-based automated AG reasoning paradigm has been extended to check simulation [5] and 
deadlock [6]. Alur et al. [1], and Sinha et al. [21], have investigated symbolic and lazy SAT-based implementations, respectively. Tsay and Wang [22] show that verification of safety properties of $\infty$-regular systems is reducible the standard AG framework. In contrast, our focus is on the verification of arbitrary $\omega$-regular-properties of $\omega$-regular-systems.

In summary, we present a very general formalization, called LAG, of the learning-based automated AG paradigm. We instantiate LAG to verify $\omega$-regular properties of reactive systems with $\omega$-regular behavior. We also show how existing approaches for automated AG reasoning are special instances of LAG. In addition, we prove the soundness and completeness of circular and non-circular AG proof rules in the context of $\omega$-regular languages. Recently, techniques to reduce the number of queries [7], and refine the assumption alphabet [11], have been proposed in the context of using automated AG to verify safety properties. We believe that these techniques are applicable for $\omega$-regular-properties as well.

\section{References}

[1] R. Alur, P. Madhusudan, and W. Nam. Symbolic Compositional Verification by Learning Assumptions. In Procs. of CAV'05, volume 3576 of LNCS, pages 548-562. Springer, July 2005.

[2] D. Angluin. Learning Regular Sets from Queries and Counterexamples. Inf. Comput., 75(2):87-106, 1987.

[3] H. Barringer, D. Giannakopoulou, and C. S. Păsăreanu. Proof Rules for Automated Compositional Verification Through Learning. In Procs. of SAVCBS ’03, pages 14-21, Sept. 2003.

[4] H. Calbrix, M. Nivat, and A. Podelski. Ultimately Periodic Words of Rational $\omega$-Languages. In Proc. of MPFS'93, 1993.

[5] S. Chaki, E. M. Clarke, N. Sinha, and P. Thati. Automated Assume-Guarantee Reasoning for Simulation Conformance. In Procs. of CAV '05, volume 3576 of LNCS, pages 534-547. Springer, July 2005.

[6] S. Chaki and N. Sinha. Assume-Guarantee Reasoning for Deadlock. In Procs. of FMCAD '06.

[7] S. Chaki and O. Strichman. Optimized L* for Assume-Guarantee Reasoning. In Procs. of TACAS '07.

[8] E. Clarke, D. Long, and K. McMillan. Compositional Model Checking. In Procs. of LICS '89.

[9] J. M. Cobleigh, D. Giannakopoulou, and C. S. Păsăreanu. Learning Assumptions for Compositional Verification. In Procs. of TACAS '03, volume 2619 of LNCS, pages 331-346. Springer, Apr. 2003.

[10] A. Farzan, Y. Chen, E. Clarke, Y. Tsan, and B. Wang. Extending Automated Compositional Verification to the Full Class of Omega-Regular Languages. In Procs. of TACAS '08. Springer, 2008.

[11] M. Gheorghiu, D. Giannakopoulou, and C. S. Păsăreanu. Refining Interface Alphabets for Compositional Verification. In Procs. of TACAS '07, volume 4424 of LNCS, pages 292-307. Springer, Mar. 2007.

[12] E. M. Gold. Language Identification in the Limit. Information and Control, 10(5):447-474, May 1967.

[13] O. Grumberg and D. Long. Model Checking and Modular Verification. TOPLAS, 16(3):843-871, May 1994.

[14] T. A. Henzinger, S. Qadeer, and S. K. Rajamani. Decomposing Refinement Proofs Using Assume-Guarantee Reasoning. In Procs. of ICCAD ’00, pages 245-252. IEEE, Nov. 2000.

[15] C. A. R. Hoare. Communicating Sequential Processes. Prentice Hall, 1985.

[16] C. B. Jones. Specification and Design of (Parallel) Programs. In Proceedings of the 9th IFIP World Congress, volume 83 of Information Processing, pages 321-332, September 1983.

[17] O. Maler and A. Pnueli. On the Learnability of Infinitary Regular Sets. Inf. Comput., 118(2):316-326, 1995.

[18] J. Misra and K. M. Chandy. Proofs of Networks of Processes. TSE, 7(4):417-426, July 1981.

[19] A. Pnueli. In Transition from Global to Modular Temporal Reasoning About Programs. Logics and Models of Concurrent Systems, 13:123-144, 1985.

[20] R. Rivest and R. Schapire. Inference of Finite Automata Using Homing Sequences. Inf. Comput., $103,1993$.

[21] N. Sinha and E. Clarke. SAT-based Compositional Verification Using Lazy Learning. In Procs. of CAV '07.

[22] Y.-K. Tsay and B.-Y. Wang. Automated Compositional Reasoning of Intuitionistically Closed Regular Properties. In Procs. of CIAA'08, pages 36-45, 2008.

[23] B.-Y. Wang. Automatic Derivation of Compositional Rules in Automated Compositional Reasoning. In Procs. of CONCUR'07, pages 303-316, 2007. 